\title{
Third-Order Inference for the Weibull Distribution
}

\author{
M. Rekkas* A. Wong ${ }^{\dagger}$
}

\begin{abstract}
Recent third-order likelihood analysis theory is applied to obtain improved confidence intervals for scalar component interest parameters of the Weibull model. This theory involves implicit but appropriate conditioning and marginalization. Both the censored and complete Weibull models are examined where primary interest is focussed on assessing the shape, scale or median parameter. The simulation results indicate the increased accuracy of this method relative to the conventional first-order likelihood ratio and Wald departure procedures. Applications to strike duration data are presented.
\end{abstract}

Keywords: Likelihood; Conditioning; Weibull distribution

${ }^{*}$ Corresponding author. Department of Economics, Simon Fraser University, 8888 University Drive, Burnaby, British Columbia V5A 1S6, email: mrekkas@sfu.ca, phone: (604) 268-6793, fax: (604) 291-5944

${ }^{\dagger}$ SASIT, York University, 4700 Keele Street, Toronto, Ontario M3J 1P3. 


\section{Introduction}

The Weibull distribution has been widely used in the empirical modelling of economic models. Applications include the modelling of unemployment spells, strike durations, income distributions, the length of a firm's innovation period, and the size of research and development budgets. Depending on the particular problem, the variable under consideration may not be fully observed, requiring censoring procedures for estimation.

Confidence intervals for testing the parameters of the Weibull model are typically constructed using maximum likelihood estimates which are asymptotically normally distributed. These confidence intervals have first-order accuracy and are computationally simple and readily accessible from statistical packages. However, these intervals have been found to perform quite poorly in terms of coverage probabilities. As an alternative, first-order confidence intervals based on likelihood ratio tests have been examined, and while computationally more costly, they have been found to perform relatively well in certain cases. Considerable scope for improvement nonetheless remains.

In this paper, recent likelihood analysis theory is used to obtain highly accurate (third-order) inferences for scalar interest parameters of the Weibull distribution. The asymptotic theory developed by Fraser and Reid (1995) is based on the likelihood structure of the data and involves conditioning and marginalization for intrinsic measures of departure to produce third-order accurate tail approximations. The methodology provides an improvement to the results obtained using large sample normal theory and those based on the likelihood ratio test. Confidence intervals and simulations are provided for both the censored and complete cases that show the improved accuracy of this method. An example using the benchmark strike duration data provided in Kennan (1985) is examined.

The paper is structured as follows. Section 2 presents the Weibull distribution. The third-order likelihood theory is described and applied to both the censored and complete cases in Section 3. Applications and simulations are contained in Section 4. Section 5 concludes and gives directions for future research.

\section{The Weibull Distribution}

The Weibull probability density function for the random variable $X$ is given by

$$
f(x ; \beta, \eta)=\frac{\beta}{\eta}\left(\frac{x}{\eta}\right)^{\beta-1} \exp \left\{-\left(\frac{x}{\eta}\right)^{\beta}\right\} \quad x>0,
$$

where $\beta>0$ and $\eta>0$ are the shape and scale parameters, respectively. Writing

$$
W=\mu+\sigma Y
$$


where $Y$ is a random variable with standardized extreme value density,

$$
f(y)=\exp \left\{y-e^{y}\right\} \quad-\infty<y<\infty
$$

then the density for $W$ can be obtained as

$$
f(w ; \mu, \sigma)=\frac{1}{\sigma} \exp \left\{\frac{w-\mu}{\sigma}-\exp \left(\frac{w-\mu}{\sigma}\right)\right\} \quad-\infty<w<\infty .
$$

The distribution of $W$ is known as the Smallest Extreme Value Distribution (SEV). If $W=\log X$, so that, $X=e^{W}$, then

$$
f(x ; \mu, \sigma)=\frac{1}{x} \frac{1}{\sigma}\left(x e^{-\mu}\right)^{\frac{1}{\sigma}} \exp \left\{-\left(x e^{-\mu}\right)^{\frac{1}{\sigma}}\right\}
$$

With $\sigma=1 / \beta$ and $\mu=\log \eta, X$ is distributed as Weibull with shape parameter $\beta$ and scale parameter $\eta$. Given this, for analytical and computational convenience, this paper works in the $W=\log X$ scale, the results, however, are reported directly for the Weibull parameters. In addition to the shape and scale parameters, there is typically an interest in inference regarding the $\alpha^{\text {th }}$ percentile. This quantity is calculated as

$$
w_{\alpha}=\log \left\{\eta[-\log (1-\alpha)]^{1 / \beta}\right\}
$$

Third-order likelihood analysis results for model (2) are provided below assuming a pre-specified censoring point. The complete data model is easily handled as a special case.

\section{Third-Order Likelihood Analysis}

\subsection{Censored Case}

Let $w=\left(w_{1}, \ldots, w_{n}\right)$ be a random sample from the SEV distribution given in (2). Here, $r$ observations with ranked data are observed, $w_{1} \leq w_{2} \leq \ldots \leq w_{r} \leq w_{c}$, where $w_{c}$ represents the censoring point, that is, $(n-r)$ observations survive beyond $w_{c}$. See Lawless (1982) for details. The log-likelihood function of $\theta=(\mu, \sigma)$ is given by

$$
l(\theta)=-r \log \sigma+\sum_{i=1}^{r}\left\{\frac{w_{i}-\mu}{\sigma}-\exp \left\{\frac{w_{i}-\mu}{\sigma}\right\}\right\}-(n-r) \exp \left\{\frac{w_{c}-\mu}{\sigma}\right\}
$$

with derivatives

$$
\begin{aligned}
& l_{\mu}(\theta)=\sum_{i=1}^{r}\left\{-\frac{1}{\sigma}+\frac{1}{\sigma} \exp \left\{\frac{w_{i}-\mu}{\sigma}\right\}\right\}+(n-r) \frac{1}{\sigma} \exp \left\{\frac{w_{c}-\mu}{\sigma}\right\} \\
& l_{\sigma}(\theta)=-\frac{r}{\sigma}+\sum_{i=1}^{r}\left\{-\frac{w_{i}-\mu}{\sigma^{2}}+\frac{w_{i}-\mu}{\sigma^{2}} \exp \left\{\frac{w_{i}-\mu}{\sigma}\right\}\right\}+(n-r) \frac{w_{c}-\mu}{\sigma^{2}} \exp \left\{\frac{w_{c}-\mu}{\sigma}\right\} .
\end{aligned}
$$


The normal equations are solved to obtain the maximum likelihood estimate $\hat{\theta}=(\hat{\mu}, \hat{\sigma})$. The information matrix denoted, $j_{\theta \theta}(\theta)$, is calculated as

$$
j_{\theta \theta}(\theta)=\left[\begin{array}{cc}
-l_{\mu \mu}(\theta) & -l_{\mu \sigma}(\theta) \\
-l_{\mu \sigma}(\theta) & -l_{\sigma \sigma}(\theta)
\end{array}\right]=\left[\begin{array}{cc}
j_{\mu \mu}(\theta) & j_{\mu \sigma}(\theta) \\
j_{\mu \sigma}(\theta) & j_{\sigma \sigma}(\theta)
\end{array}\right],
$$

where

$$
\begin{aligned}
l_{\mu \mu}(\theta)= & \sum_{i=1}^{r}\left\{-\frac{1}{\sigma^{2}} \exp \left\{\frac{w_{i}-\mu}{\sigma}\right\}\right\}-(n-r) \frac{1}{\sigma^{2}} \exp \left\{\frac{w_{c}-\mu}{\sigma}\right\} \\
l_{\mu \sigma}(\theta)= & \sum_{i=1}^{r}\left\{\frac{1}{\sigma^{2}}-\frac{1}{\sigma^{2}} \exp \left\{\frac{w_{i}-\mu}{\sigma}\right\}-\frac{w_{i}-\mu}{\sigma^{3}} \exp \left\{\frac{w_{i}-\mu}{\sigma}\right\}\right\}- \\
& (n-r) \frac{1}{\sigma^{2}} \exp \left\{\frac{w_{c}-\mu}{\sigma}\right\}-(n-r) \frac{w_{c}-\mu}{\sigma^{3}} \exp \left\{\frac{w_{c}-\mu}{\sigma}\right\} \\
l_{\sigma \sigma}(\theta)= & \frac{r}{\sigma^{2}}+\sum_{i=1}^{r}\left\{\frac{2\left(w_{i}-\mu\right)}{\sigma^{3}}-\frac{2\left(w_{i}-\mu\right)}{\sigma^{3}} \exp \left\{\frac{w_{i}-\mu}{\sigma}\right\}-\frac{\left(w_{i}-\mu\right)^{2}}{\sigma^{4}} \exp \left\{\frac{w_{i}-\mu}{\sigma}\right\}\right\}- \\
& (n-r) \frac{2\left(w_{c}-\mu\right)}{\sigma^{3}} \exp \left\{\frac{w_{c}-\mu}{\sigma}\right\}-(n-r) \frac{\left(w_{c}-\mu\right)^{2}}{\sigma^{4}} \exp \left\{\frac{w_{c}-\mu}{\sigma}\right\} .
\end{aligned}
$$

The observed information matrix is obtained as

$$
j_{\theta \theta}(\hat{\theta})=\left[\begin{array}{cc}
j_{\mu \mu}(\hat{\theta}) & j_{\mu \sigma}(\hat{\theta}) \\
j_{\mu \sigma}(\hat{\theta}) & j_{\sigma \sigma}(\hat{\theta})
\end{array}\right]
$$

where $j_{\mu \mu}(\hat{\theta})=-l_{\mu \mu}(\hat{\theta}), j_{\mu \sigma}(\hat{\theta})=-l_{\mu \sigma}(\hat{\theta})$, and $j_{\sigma \sigma}(\hat{\theta})=-l_{\sigma \sigma}(\hat{\theta})$. Familiar first-order asymptotic methods can be used to test the null hypothesis of $\mu=\mu_{0}$. The standardized maximum likelihood departure and signed square root of the log likelihood ratio statistic are given respectively by

$$
\begin{aligned}
q & =\left(\hat{\mu}-\hat{\mu}_{0}\right)\left\{j^{\mu \mu}(\hat{\theta})\right\}^{-1 / 2} \\
r & =\operatorname{sgn}\left(\hat{\mu}-\mu_{0}\right)\left\{2\left[l(\hat{\theta})-l\left(\hat{\theta}_{\mu_{0}}\right)\right]\right\}^{1 / 2}
\end{aligned}
$$

where $\hat{\theta}_{\mu_{0}}=\left(\mu_{0}, \hat{\sigma}_{\mu_{0}}\right)$ is the constrained maximum likelihood estimate of $\theta$ at $\mu_{0}$ and $j^{\mu \mu}(\hat{\theta})$ is the appropriate entry from the estimated asymptotic variance matrix of $\hat{\theta}$

$$
j^{\theta \theta}(\hat{\theta})=\left[\begin{array}{cc}
j^{\mu \mu}(\hat{\theta}) & j^{\mu \sigma}(\hat{\theta}) \\
j^{\mu \sigma}(\hat{\theta}) & j^{\sigma \sigma}(\hat{\theta})
\end{array}\right] .
$$

The quantities $q$ and $r$ have the standard normal as their limiting distribution and thus tail probabilities for testing the null can be approximated by $\Phi(q)$ and $\Phi(r)$ respectively, where $\Phi(\cdot)$ is the cumulative distribution function of the standard normal distribution.

Recent third-order likelihood methods developed by Fraser and Reid (1995) are applied to obtain highly accurate inferences for any scalar component parameter of interest of the full-dimensional 
model. The theory involves the construction of a particular maximum likelihood departure, $Q$, that is used together with the signed square root of the log-likelihood ratio (5) to produce third-order approximations to the left tail probabilities for testing scalar interest parameters. Explicit nuisance parameterization is not required for this method. Details are given in Fraser et al. (1999).

There are two fundamental steps involved in this likelihood analysis theory. The first involves a dimension reduction from the dimension of the variable to the dimension of the parameter. This dimension reduction is achieved by conditioning on an implicit ancillary statistic. In fact, only tangent directions, $V$, to this ancillary statistic are necessary. The resulting model is then approximated by a tangent exponential model with the canonical parameter involving the conditioning vectors as well as derivatives of the log-likelihood function with respect to the data. The second step involves a further dimension reduction through marginalization which reduces the dimension of the exponential model to the desired dimension of one for testing the null hypothesis of $\psi(\theta)=\psi_{0}$. A review of the necessary technical components resulting from this theory and the third-order tail approximations used in the analysis of the Weibull model are given below.

Maximization of the Lagrangean, $H(\theta)$, is used to obtain the constrained maximum likelihood estimate $\hat{\theta}_{\psi_{0}}$

$$
\max _{\theta, \lambda} H(\theta)=l(\theta)+\lambda\left(\psi(\theta)-\psi_{0}\right) .
$$

Setting the first-order conditions

$$
\begin{aligned}
& H_{\mu}(\theta)=l_{\mu}(\theta)+\lambda \psi_{\mu}(\theta) \\
& H_{\sigma}(\theta)=l_{\sigma}(\theta)+\lambda \psi_{\sigma}(\theta) \\
& H_{\lambda}(\theta)=\psi(\theta)-\psi_{0}
\end{aligned}
$$

equal to zero and solving the system of equations yields the constrained maximum likelihood solution for the parameter $\theta$ and the Lagrange multiplier $\lambda$. Note that $l_{\mu}(\theta)=\partial l(\theta) / \partial \mu$ and $\psi_{\mu}(\theta)=\partial \psi(\theta) / \partial \mu$. The tilted log-likelihood is defined as the quantity

$$
\tilde{l}(\theta)=l(\theta)+\tilde{\lambda}\left(\psi(\theta)-\psi_{0}\right)
$$

where $\tilde{\lambda}$, the Lagrange multiplier solution, is substituted into the Lagrangean, $H(\theta)$. A nominal information matrix is given by

$$
\tilde{\jmath}_{\theta \theta}\left(\hat{\theta}_{\psi_{0}}\right)=-\tilde{l}_{\theta \theta}\left(\hat{\theta}_{\psi_{0}}\right)
$$

Fraser and Reid (1995) show that the tangent vectors, $V$, to the ancillary statistic can be obtained by using a pivotal quantity, $k$, and differentiating the data, $w$, with respect to the full parameter, $\theta$, 
while holding the pivotal quantity fixed. The vectors are calculated as follows:

$$
V=\left.\frac{\partial w}{\partial \theta}\right|_{\hat{\theta}}=\left.\left\{\frac{\partial k(w, \theta)}{\partial w}\right\}^{-1}\left\{\frac{\partial k(w, \theta)}{\partial \theta}\right\}\right|_{\hat{\theta}} .
$$

These vectors are used to calculate the local canonical parameter

$$
\varphi(\theta)=\frac{\partial l(\theta)}{\partial w} V
$$

This locally defined canonical parameter then becomes the relevant parameter space. Given this new parameterization, a marginalization procedure gives the recalibrated interest parameter

$$
\chi(\theta)=\psi_{\theta}\left(\hat{\theta}_{\psi_{0}}\right) \varphi_{\theta}^{-1}\left(\hat{\theta}_{\psi_{0}}\right) \varphi(\theta)
$$

where $\chi(\theta)=\psi(\theta)$ in the $\varphi(\theta)$ scale and $\varphi_{\theta}(\theta)=\partial \varphi(\theta) / \partial \theta$. Additionally, the estimate of the variance of $\chi(\theta)$ is given by

$$
\hat{\sigma}_{\chi}^{2}=\psi_{\theta}\left(\hat{\theta}_{\psi_{0}}\right) \tilde{\jmath}^{\theta \theta}\left(\hat{\theta}_{\psi_{0}}\right) \psi_{\theta}^{\prime}\left(\hat{\theta}_{\psi_{0}}\right)
$$

The standardized maximum likelihood departure in the $\varphi(\theta)$ scale is

$$
Q=\operatorname{sgn}\left(\hat{\psi}-\psi_{0}\right)\left|\chi(\hat{\theta})-\chi\left(\hat{\theta}_{\psi_{0}}\right)\right|\left\{\frac{\left|j_{(\theta \theta)}(\hat{\theta})\right|}{\hat{\sigma}_{\chi}^{2}\left|\tilde{J}_{(\theta \theta)}\left(\hat{\theta}_{\psi_{0}}\right)\right|}\right\},
$$

where the recalibrated determinants are calculated as

$$
\begin{aligned}
\left|j_{(\theta \theta)}(\hat{\theta})\right| & =\left|j_{\theta \theta}(\hat{\theta})\right|\left|\varphi_{\theta}(\hat{\theta})\right|^{-2} \\
\left|\tilde{\jmath}_{(\theta \theta)}\left(\hat{\theta}_{\psi_{0}}\right)\right| & =\left|\tilde{\jmath}_{\theta \theta}\left(\hat{\theta}_{\psi_{0}}\right)\right|\left|\varphi_{\theta}\left(\hat{\theta}_{\psi_{0}}\right)\right|^{-2} .
\end{aligned}
$$

The determinants in the above expressions involving the $\varphi_{\theta}(\hat{\theta})$ and $\varphi_{\theta}\left(\hat{\theta}_{\psi_{0}}\right)$ terms can be thought of as Jacobian terms. Two asymptotically equivalent third-order approximations to tail probabilities are given by Lugannani and Rice (1980)

$$
\Phi(R)+\phi(R)\left\{\frac{1}{R}-\frac{1}{Q}\right\}
$$

and Barndorff-Nielsen (1991)

$$
\Phi\left(R^{*}\right),
$$

where $\phi(\cdot)$ is the standard normal density function, $R$ is the modified signed log-likelihood ratio statistic given by

$$
\begin{aligned}
R & =\operatorname{sgn}\left(\hat{\psi}-\psi_{0}\right)\left\{2\left[l(\hat{\theta})-l\left(\hat{\theta}_{\psi_{0}}\right)\right]\right\}^{1 / 2} \\
& =\operatorname{sgn}\left(\hat{\psi}-\psi_{0}\right)\left\{2\left[l(\hat{\theta})-\tilde{l}\left(\hat{\theta}_{\psi_{0}}\right)\right]\right\}^{1 / 2}
\end{aligned}
$$


and $R^{*}$ is the modified signed log-likelihood ratio statistic given by

$$
R^{*}=R-R^{-1} \log \left(\frac{R}{Q}\right) .
$$

The equality from (16) to (17) follows directly from the optimality conditions.

The complexity of the third-order method lies in the calculation of the maximum likelihood departure measure, $Q$, given by (11). Once this quantity has been obtained, with a large portion of its components likewise required for the computation of the likelihood ratio and mle-based intervals, the complexity of the computations does not increase with sample size.

\subsubsection{Interest Parameters}

Three scalar component interest parameters for the censored model given in (3) are considered: the shape parameter, $\beta=1 / \sigma$, the scale parameter, $\eta=e^{\mu}$, and the $\alpha^{t h}$ percentile, $x_{\alpha}=e^{\mu}[-\log (1-\alpha)]^{\sigma} \Leftrightarrow$ $w_{\alpha}=\log \left\{\eta[-\log (1-\alpha)]^{1 / \beta}\right\}$. Making use of the 1-1 relationships, the following three cases are considered.

Case 1: $\psi=\sigma$

The constrained maximum likelihood estimate for this case is $\psi_{0}=\sigma_{0}$. The Lagrangean is

$$
H(\theta)=l(\theta)+\lambda\left(\sigma-\sigma_{0}\right)
$$

and differentiation yields

$$
\begin{aligned}
& H_{\mu}(\theta)=l_{\mu}(\theta) \\
& H_{\sigma}(\theta)=l_{\sigma}(\theta)+\lambda \\
& H_{\lambda}(\theta)=\sigma-\sigma_{0} .
\end{aligned}
$$

The constrained maximum likelihood estimates satisfy

$$
\begin{aligned}
l_{\mu}(\tilde{\theta}) & =0 \\
\tilde{\lambda} & =-l_{\sigma}(\tilde{\theta}) \\
\tilde{\sigma} & =\sigma_{0} .
\end{aligned}
$$

The tilted likelihood then is

$$
\tilde{l}(\theta)=l(\theta)+\tilde{\lambda}\left(\sigma-\sigma_{0}\right),
$$


with

$$
\begin{aligned}
\tilde{l}_{\mu}(\theta) & =l_{\mu}(\theta) \\
\tilde{l}_{\sigma}(\theta) & =l_{\sigma}(\theta)+\tilde{\lambda} \\
\tilde{l}_{\mu \mu}(\theta) & =l_{\mu \mu}(\theta) \\
\tilde{l}_{\mu \sigma}(\theta) & =l_{\mu \sigma}(\theta) \\
\tilde{l}_{\sigma \sigma}(\theta) & =l_{\sigma \sigma}(\theta) .
\end{aligned}
$$

Notice the above implies

$$
\tilde{\jmath}_{\theta \theta}(\tilde{\theta})=j_{\theta \theta}(\tilde{\theta})
$$

The ancillary direction vectors, $V$, are

$$
V=\left[\begin{array}{cc}
1 & \frac{w_{1}-\hat{\mu}}{\hat{\sigma}} \\
\vdots & \vdots \\
1 & \frac{w_{r}-\hat{\mu}}{\hat{\sigma}} \\
1 & \frac{w_{c}-\hat{\mu}}{\hat{\sigma}}
\end{array}\right] .
$$

These tangent directions are used to obtain the canonical parameter

$$
\varphi(\theta)=\left[\varphi_{1}(\theta) \quad \varphi_{2}(\theta)\right],
$$

where

$$
\begin{aligned}
& \varphi_{1}(\theta)=\sum_{i=1}^{r}\left\{\frac{1}{\sigma}-\frac{1}{\sigma} \exp \left\{\frac{w_{i}-\mu}{\sigma}\right\}\right\}-(n-r) \frac{1}{\sigma} \exp \left\{\frac{w_{c}-\mu}{\sigma}\right\}=-l_{\mu}(\theta) \\
& \varphi_{2}(\theta)=\sum_{i=1}^{r}\left\{\left\{\frac{1}{\sigma}-\frac{1}{\sigma} \exp \left\{\frac{w_{i}-\mu}{\sigma}\right\}\right\} \frac{w_{i}-\hat{\mu}}{\hat{\sigma}}\right\}-(n-r) \frac{1}{\sigma} \exp \left\{\frac{w_{c}-\mu}{\sigma}\right\} \frac{w_{c}-\hat{\mu}}{\hat{\sigma}} .
\end{aligned}
$$

The parameter $\chi(\theta)$ is then obtained as

$$
\chi(\theta)=\psi_{\theta}(\tilde{\theta}) \varphi_{\theta}^{-1}(\tilde{\theta}) \varphi(\theta)
$$

with $\psi_{\theta}(\theta)=\left[\begin{array}{ll}\psi_{\mu}(\theta) & \psi_{\sigma}(\theta)\end{array}\right]=\left[\begin{array}{ll}0 & 1\end{array}\right]$ and $\varphi_{\theta}^{-1}(\theta)$ is calculated from

$$
\varphi_{\theta}(\theta)=\left[\begin{array}{ll}
\varphi_{1 \mu}(\theta) & \varphi_{1 \sigma}(\theta) \\
\varphi_{2 \mu}(\theta) & \varphi_{2 \sigma}(\theta)
\end{array}\right],
$$

where

$$
\varphi_{1 \mu}(\theta)=-l_{\mu \mu}(\theta)=j_{\mu \mu}(\theta)
$$




$$
\begin{aligned}
\varphi_{1 \sigma}(\theta)= & -l_{\mu \sigma}(\theta)=j_{\mu \sigma}(\theta) \\
\varphi_{2 \mu}(\theta)= & \sum_{i=1}^{r} \frac{1}{\sigma^{2}} \exp \left\{\frac{w_{i}-\mu}{\sigma}\right\} \frac{w_{i}-\hat{\mu}}{\hat{\sigma}}+(n-r) \frac{1}{\sigma^{2}} \exp \left\{\frac{w_{c}-\mu}{\sigma}\right\} \frac{w_{c}-\hat{\mu}}{\hat{\sigma}} \\
\varphi_{2 \sigma}(\theta)= & \sum_{i=1}^{r}\left[-\frac{1}{\sigma^{2}}+\frac{1}{\sigma^{2}} \exp \left\{\frac{w_{i}-\mu}{\sigma}\right\}+\frac{w_{i}-\mu}{\sigma^{3}} \exp \left\{\frac{w_{i}-\mu}{\sigma}\right\}\right] \frac{w_{i}-\hat{\mu}}{\hat{\sigma}}+ \\
& (n-r) \frac{1}{\sigma^{2}} \exp \left\{\frac{w_{c}-\mu}{\sigma}\right\} \frac{w_{c}-\hat{\mu}}{\hat{\sigma}}+(n-r) \frac{w_{c}-\mu}{\sigma^{3}} \exp \left\{\frac{w_{c}-\mu}{\sigma}\right\} \frac{w_{i}-\hat{\mu}}{\hat{\sigma}} .
\end{aligned}
$$

As before

$$
\hat{\sigma}_{\chi}^{2}=\psi_{\theta}(\tilde{\theta}) \tilde{\jmath}^{\theta \theta}(\tilde{\theta}) \psi_{\theta}(\tilde{\theta})
$$

where $\tilde{\jmath}^{\theta \theta}(\tilde{\theta})$ is the estimated asymptotic covariance matrix of $\tilde{\theta}$. The signed likelihood ratio departure is

$$
R=\operatorname{sgn}\left(\hat{\psi}-\psi_{0}\right)\{2[l(\hat{\theta})-l(\tilde{\theta})]\}^{1 / 2}
$$

and the maximum likelihood departure is

$$
Q=\operatorname{sgn}\left(\hat{\psi}-\psi_{0}\right)|\chi(\hat{\theta})-\chi(\tilde{\theta})|\left\{\frac{\left|\hat{\jmath}_{\theta \theta}(\hat{\theta})\right|\left|\varphi_{\theta}(\hat{\theta})\right|^{-2}}{\left.\hat{\sigma}_{\chi}^{2}\left|\tilde{\jmath}_{\theta \theta}(\tilde{\theta})\right| \varphi_{\theta}(\tilde{\theta})\right|^{-2}}\right\}^{1 / 2},
$$

with the recalibrated determinants calculated as in (12) and (13). Tail probabilities can be calculated using either the Lugannani and Rice or Barndorff-Nielsen formulas.

Case 2: $\psi=\mu$

The Lagrangean for this case is

$$
H(\theta)=l(\theta)+\lambda\left(\mu-\mu_{0}\right)
$$

Differentiation with respect to the parameters and the Lagrange multiplier leads to

$$
\begin{aligned}
& H_{\mu}(\theta)=l_{\mu}(\theta)+\lambda \\
& H_{\sigma}(\theta)=l_{\sigma}(\theta) \\
& H_{\lambda}(\theta)=\mu-\mu_{0} .
\end{aligned}
$$

The constrained maximum likelihood estimates satisfy

$$
\begin{aligned}
\tilde{\lambda} & =-l_{\mu}(\tilde{\theta}) \\
l_{\sigma}(\tilde{\theta}) & =0 \\
\tilde{\mu} & =\mu_{0} .
\end{aligned}
$$


The tilted log-likelihood is

$$
\tilde{l}=l(\theta)+\tilde{\lambda}\left(\mu-\mu_{0}\right)
$$

with

$$
\begin{aligned}
\tilde{l}_{\mu}(\theta) & =l_{\mu}(\theta)+\tilde{\lambda} \\
\tilde{l}_{\sigma}(\theta) & =l_{\sigma}(\theta) \\
\tilde{l}_{\mu \mu}(\theta) & =l_{\mu \mu}(\theta) \\
\tilde{l}_{\mu \sigma}(\theta) & =l_{\mu \sigma}(\theta) \\
\tilde{l}_{\sigma \sigma}(\theta) & =l_{\sigma \sigma}(\theta) .
\end{aligned}
$$

The rest of the quantities are the same as in Case 1 with $\psi_{\theta}(\theta)=\left[\begin{array}{ll}1 & 0\end{array}\right]$.

Case 3: $\psi=w_{\alpha}$

The interest parameter $\psi$ can be expressed as $\psi=\mu+\sigma A$ where $A=\log [-\log (1-\alpha)]$ and $w_{\alpha}$ is the $\alpha^{\text {th }}$ percentile. The Lagrangean for this case is

$$
H(\theta)=l(\theta)+\lambda\left(\mu+\sigma A-\psi_{0}\right)
$$

Differentiation leads to

$$
\begin{aligned}
& H_{\mu}(\theta)=l_{\mu}(\theta)+\lambda \\
& H_{\sigma}(\theta)=l_{\sigma}(\theta)+\lambda A \\
& H_{\lambda}(\theta)=\mu+\sigma A-\psi_{0} .
\end{aligned}
$$

The constrained maximum likelihood estimates satisfy

$$
\begin{aligned}
\tilde{\lambda} & =-l_{\mu}(\tilde{\theta}) \\
l_{\sigma}(\tilde{\theta}) & =A l_{\mu}(\tilde{\theta}) \\
\tilde{\mu} & =\psi_{0}-A \tilde{\sigma} .
\end{aligned}
$$

The tilted log-likelihood is

$$
\tilde{l}=l(\theta)+\tilde{\lambda}\left(\mu+\sigma A-\psi_{0}\right),
$$

with

$$
\tilde{l}_{\mu}(\theta)=l_{\mu}(\theta)+\tilde{\lambda}
$$




$$
\begin{aligned}
\tilde{l}_{\sigma}(\theta) & =l_{\sigma}(\theta)+\tilde{\lambda} A \\
\tilde{l}_{\mu \mu}(\theta) & =l_{\mu \mu}(\theta) \\
\tilde{l}_{\mu \sigma}(\theta) & =l_{\mu \sigma}(\theta) \\
\tilde{l}_{\sigma \sigma}(\theta) & =l_{\sigma \sigma}(\theta) .
\end{aligned}
$$

The rest of the quantities are likewise the same as in Case 1 with $\psi_{\theta}(\theta)=\left[\begin{array}{ll}1 & A\end{array}\right]$.

\subsection{Complete Case}

In the absence of censoring, the special complete data case $(r=n)$ results. For the random sample $w=\left(w_{1}, \ldots, w_{n}\right)$ from the SEV distribution, the log-likelihood function for $\theta=(\mu, \sigma)$ is adjusted as follows

$$
l(\theta)=-n \log \sigma+\sum_{i=1}^{n}\left\{\frac{w_{i}-\mu}{\sigma}-\exp \left\{\frac{w_{i}-\mu}{\sigma}\right\}\right\}
$$

with derivatives

$$
\begin{aligned}
& l_{\mu}(\theta)=\sum\left\{-\frac{1}{\sigma}+\frac{1}{\sigma} \exp \left\{\frac{w_{i}-\mu}{\sigma}\right\}\right\} \\
& l_{\sigma}(\theta)=-\frac{n}{\sigma}+\sum\left\{-\frac{w_{i}-\mu}{\sigma^{2}}+\frac{w_{i}-\mu}{\sigma^{2}} \exp \left\{\frac{w_{i}-\mu}{\sigma}\right\}\right\} .
\end{aligned}
$$

The normal equations are solved to obtain the maximum likelihood estimate $\hat{\theta}=(\hat{\mu}, \hat{\sigma})$. In particular, $\hat{\theta}$ simultaneously satisfies

$$
\begin{array}{r}
\sum \exp \left\{\frac{w_{i}-\hat{\mu}}{\hat{\sigma}}\right\}=n \\
\sum\left\{\frac{w_{i}-\hat{\mu}}{\hat{\sigma}} \exp \left\{\frac{w_{i}-\hat{\mu}}{\hat{\sigma}}\right\}-\frac{w_{i}-\hat{\mu}}{\hat{\sigma}}\right\}=n .
\end{array}
$$

The information matrix denoted, $j_{\theta \theta}(\theta)$, is calculated as

$$
j_{\theta \theta}(\theta)=\left[\begin{array}{cc}
-l_{\mu \mu}(\theta) & -l_{\mu \sigma}(\theta) \\
-l_{\mu \sigma}(\theta) & -l_{\sigma \sigma}(\theta)
\end{array}\right]=\left[\begin{array}{cc}
j_{\mu \mu}(\theta) & j_{\mu \sigma}(\theta) \\
j_{\mu \sigma}(\theta) & j_{\sigma \sigma}(\theta)
\end{array}\right],
$$

where

$$
\begin{aligned}
& l_{\mu \mu}(\theta)=\sum\left\{-\frac{1}{\sigma^{2}} \exp \left\{\frac{w_{i}-\mu}{\sigma}\right\}\right\} \\
& l_{\mu \sigma}(\theta)=\sum\left\{\frac{1}{\sigma^{2}}-\frac{1}{\sigma^{2}} \exp \left\{\frac{w_{i}-\mu}{\sigma}\right\}-\frac{w_{i}-\mu}{\sigma^{3}} \exp \left\{\frac{w_{i}-\mu}{\sigma}\right\}\right\} \\
& l_{\sigma \sigma}(\theta)=\frac{n}{\sigma^{2}}+\sum\left\{\frac{2\left(w_{i}-\mu\right)}{\sigma^{3}}-\frac{2\left(w_{i}-\mu\right)}{\sigma^{3}} \exp \left\{\frac{w_{i}-\mu}{\sigma}\right\}-\frac{\left(w_{i}-\mu\right)^{2}}{\sigma^{4}} \exp \left\{\frac{w_{i}-\mu}{\sigma}\right\}\right\} .
\end{aligned}
$$

The observed information matrix is obtained as

$$
j_{\theta \theta}(\hat{\theta})=\left[\begin{array}{cc}
j_{\mu \mu}(\hat{\theta}) & j_{\mu \sigma}(\hat{\theta}) \\
j_{\mu \sigma}(\hat{\theta}) & j_{\sigma \sigma}(\hat{\theta})
\end{array}\right],
$$


where

$$
\begin{aligned}
& j_{\mu \mu}(\hat{\theta})=\frac{n}{\hat{\sigma}^{2}} \\
& j_{\mu \sigma}(\hat{\theta})=\frac{1}{\hat{\sigma}^{2}} \sum \frac{w_{i}-\hat{\mu}}{\hat{\sigma}} \exp \left\{\frac{w_{i}-\hat{\mu}}{\hat{\sigma}}\right\} \\
& j_{\sigma \sigma}(\hat{\theta})=\frac{n}{\hat{\sigma}^{2}}+\frac{1}{\hat{\sigma}^{2}} \sum\left(\frac{w_{i}-\hat{\mu}}{\hat{\sigma}}\right)^{2} \exp \left\{\frac{w_{i}-\hat{\mu}}{\hat{\sigma}}\right\} .
\end{aligned}
$$

The ancillary directions are

$$
V=\left[\begin{array}{ll}
1 & \frac{w-\hat{\mu}}{\hat{\sigma}}
\end{array}\right]
$$

where the 1 represents the $n$-dimensional unit vector and the $(w-\hat{\mu}) / \hat{\sigma}$ is the $n$-dimensional standardized residual vector

$$
\left[\frac{w_{1}-\hat{\mu}}{\hat{\sigma}}, \ldots, \frac{w_{n}-\hat{\mu}}{\hat{\sigma}}\right]
$$

Given these quantities the third-order computations are conceptually similar to those for the uncensored case, and as such, the details are not provided here.

\subsubsection{Interest Parameters}

The same three scalar component interest parameters for the complete Weibull model are considered: the shape parameter, $\beta=1 / \sigma$, the scale parameter, $\eta=e^{\mu}$, and the $\alpha^{\text {th }}$ percentile, $x_{\alpha}=e^{\mu}[-\log (1-\alpha)]^{\sigma} \Leftrightarrow w_{\alpha}=\log \left\{\eta[-\log (1-\alpha)]^{1 / \beta}\right\}$.

Case 1: $\psi=\sigma$

The constrained maximum likelihood estimate for this case is then $\psi_{0}=\sigma_{0}$. The Lagrangean is

$$
H(\theta)=l(\theta)+\lambda\left(\sigma-\sigma_{0}\right)
$$

and differentiation yields

$$
\begin{aligned}
& H_{\mu}(\theta)=l_{\mu}(\theta) \\
& H_{\sigma}(\theta)=l_{\sigma}(\theta)+\lambda \\
& H_{\lambda}(\theta)=\sigma-\sigma_{0} .
\end{aligned}
$$

The constrained maximum likelihood estimates are solved as

$$
\begin{aligned}
\tilde{\sigma} & =\sigma_{0} \\
\tilde{\mu} & =\tilde{\sigma} \log \left\{\frac{1}{n} \sum \exp \left\{\frac{w_{i}}{\tilde{\sigma}}\right\}\right\} \\
\tilde{\lambda} & =\frac{n}{\tilde{\sigma}}+\sum\left\{\frac{w_{i}-\tilde{\mu}}{\tilde{\sigma}^{2}}-\frac{w_{i}-\tilde{\mu}}{\tilde{\sigma}^{2}} \exp \left\{\frac{w_{i}-\tilde{\mu}}{\tilde{\sigma}}\right\}\right\} .
\end{aligned}
$$


The tilted likelihood then is

$$
\tilde{l}(\theta)=l(\theta)+\tilde{\lambda}\left(\sigma-\sigma_{0}\right)
$$

with

$$
\begin{aligned}
\tilde{l}_{\mu}(\theta) & =l_{\mu}(\theta) \\
\tilde{l}_{\sigma}(\theta) & =l_{\sigma}(\theta)+\tilde{\lambda} \\
\tilde{l}_{\mu \mu}(\theta) & =l_{\mu \mu}(\theta) \\
\tilde{l}_{\mu \sigma}(\theta) & =l_{\mu \sigma}(\theta) \\
\tilde{l}_{\sigma \sigma}(\theta) & =l_{\sigma \sigma}(\theta) .
\end{aligned}
$$

Notice the above implies

$$
\tilde{\jmath}_{\theta \theta}(\tilde{\theta})=j_{\theta \theta}(\tilde{\theta})
$$

The ancillary direction vectors, $V$, are

$$
V=\left[\begin{array}{ll}
1 & \frac{w-\hat{\mu}}{\hat{\sigma}}
\end{array}\right]
$$

where the 1 represents the $n$-dimensional unit vector and the $w-\hat{\mu} / \hat{\sigma}$ is the $n$-dimensional standardized residual vector. These tangent directions are used to obtain the canonical parameter

$$
\varphi(\theta)=\left[\varphi_{1}(\theta) \quad \varphi_{2}(\theta)\right]
$$

where

$$
\begin{aligned}
& \varphi_{1}(\theta)=\sum\left\{\frac{1}{\sigma}-\frac{1}{\sigma} \exp \left\{\frac{w_{j}-\mu}{\sigma}\right\}\right\} \\
& \varphi_{2}(\theta)=\sum\left\{\left\{\frac{1}{\sigma}-\frac{1}{\sigma} \exp \left\{\frac{w_{j}-\mu}{\sigma}\right\}\right\} \frac{w_{j}-\hat{\mu}}{\hat{\sigma}}\right\} .
\end{aligned}
$$

The parameter $\chi(\theta)$ is then obtained as

$$
\chi(\theta)=\psi_{\theta}(\tilde{\theta}) \varphi_{\theta}^{-1}(\tilde{\theta}) \varphi(\theta)
$$

with $\psi_{\theta}(\theta)=\left[\begin{array}{ll}\psi_{\mu}(\theta) & \psi_{\sigma}(\theta)\end{array}\right]=\left[\begin{array}{ll}0 & 1\end{array}\right]$ and $\varphi_{\theta}^{-1}(\theta)$ is calculated from

$$
\varphi_{\theta}(\theta)=\left[\begin{array}{ll}
\varphi_{1 \mu}(\theta) & \varphi_{1 \sigma}(\theta) \\
\varphi_{2 \mu}(\theta) & \varphi_{2 \sigma}(\theta)
\end{array}\right],
$$


where

$$
\begin{aligned}
\varphi_{1 \mu}(\theta) & =\sum \frac{1}{\sigma^{2}} \exp \left\{\frac{w_{j}-\mu}{\sigma}\right\} \\
\varphi_{1 \sigma}(\theta) & =\sum\left[-\frac{1}{\sigma^{2}}+\frac{1}{\sigma^{2}} \exp \left\{\frac{w_{j}-\mu}{\sigma}\right\}+\frac{w_{j}-\mu}{\sigma^{3}} \exp \left\{\frac{w_{j}-\mu}{\sigma}\right\}\right] \\
\varphi_{2 \mu}(\theta) & =\sum \frac{1}{\sigma^{2}} \exp \left\{\frac{w_{j}-\mu}{\sigma}\right\} \frac{w_{j}-\hat{\mu}}{\hat{\sigma}} \\
\varphi_{2 \sigma}(\theta) & =\sum\left[-\frac{1}{\sigma^{2}}+\frac{1}{\sigma^{2}} \exp \left\{\frac{w_{j}-\mu}{\sigma}\right\}+\frac{w_{j}-\mu}{\sigma^{3}} \exp \left\{\frac{w_{j}-\mu}{\sigma}\right\}\right] \frac{w_{j}-\hat{\mu}}{\hat{\sigma}} .
\end{aligned}
$$

As before

$$
\hat{\sigma}_{\chi}^{2}=\psi_{\theta}(\tilde{\theta}) \tilde{\jmath}^{\theta \theta}(\tilde{\theta}) \psi_{\theta}(\tilde{\theta})
$$

where $\tilde{\jmath}^{\theta \theta}(\tilde{\theta})$ is the estimated asymptotic covariance matrix of $\tilde{\theta}$. The signed likelihood ratio departure is

$$
R=\operatorname{sgn}\left(\hat{\psi}-\psi_{0}\right)\{2[l(\hat{\theta})-l(\tilde{\theta})]\}^{1 / 2}
$$

and the maximum likelihood departure is

$$
Q=\operatorname{sgn}\left(\hat{\psi}-\psi_{0}\right)|\chi(\hat{\theta})-\chi(\tilde{\theta})|\left\{\frac{\left|\hat{\jmath}_{\theta \theta}(\hat{\theta})\right|\left|\varphi_{\theta}(\hat{\theta})\right|^{-2}}{\left.\hat{\sigma}_{\chi}^{2}\left|\tilde{\jmath}_{\theta \theta}(\tilde{\theta})\right| \varphi_{\theta}(\tilde{\theta})\right|^{-2}}\right\}^{1 / 2} .
$$

Tail probabilities can be calculated using either the Lugannani and Rice or Barndorff-Nielsen formulas.

Case 2: $\psi=\mu$

The Lagrangean for this case is

$$
H(\theta)=l(\theta)+\lambda\left(\mu-\mu_{0}\right)
$$

Differentiation with respect to the parameters and the Lagrange multiplier leads to

$$
\begin{aligned}
& H_{\mu}(\theta)=l_{\mu}(\theta)+\lambda \\
& H_{\sigma}(\theta)=l_{\sigma}(\theta) \\
& H_{\lambda}(\theta)=\mu-\mu_{0} .
\end{aligned}
$$

The constrained maximum likelihood estimates are solved as

$$
\begin{aligned}
\tilde{\mu} & =\mu_{0} \\
\tilde{\lambda} & =-l_{\mu}(\tilde{\theta}),
\end{aligned}
$$


where $\tilde{\sigma}$ satisfies

$$
-\frac{n}{\tilde{\sigma}}-\sum\left\{\frac{w_{i}-\tilde{\mu}}{\tilde{\sigma}^{2}}-\frac{w_{i}-\tilde{\mu}}{\tilde{\sigma}^{2}} \exp \left\{\frac{w_{i}-\tilde{\mu}}{\tilde{\sigma}}\right\}\right\}=0 .
$$

The tilted log-likelihood is

$$
\tilde{l}=l(\theta)+\tilde{\lambda}\left(\mu-\mu_{0}\right)
$$

with

$$
\begin{aligned}
\tilde{l}_{\mu}(\theta) & =l_{\mu}(\theta)+\tilde{\lambda} \\
\tilde{l}_{\sigma}(\theta) & =l_{\sigma}(\theta) \\
\tilde{l}_{\mu \mu}(\theta) & =l_{\mu \mu}(\theta) \\
\tilde{l}_{\mu \sigma}(\theta) & =l_{\mu \sigma}(\theta) \\
\tilde{l}_{\sigma \sigma}(\theta) & =l_{\sigma \sigma}(\theta) .
\end{aligned}
$$

The rest of the quantities are the same as in Case 1 with $\psi_{\theta}(\theta)=\left[\begin{array}{ll}1 & 0\end{array}\right]$.

Case 3: $\psi=w_{\alpha}$

The interest parameter $\psi$ can be expressed as $\psi=\mu+\sigma A$ where $A=\log [-\log (1-\alpha)]$ and $w_{\alpha}$ is the $\alpha^{\text {th }}$ percentile. The Lagrangean for this case is

$$
H(\theta)=l(\theta)+\lambda\left(\mu+\sigma A-\psi_{0}\right)
$$

Differentiation leads to

$$
\begin{aligned}
& H_{\mu}(\theta)=l_{\mu}(\theta)+\lambda \\
& H_{\sigma}(\theta)=l_{\sigma}(\theta)+\lambda A \\
& H_{\lambda}(\theta)=\mu+\sigma A-\psi_{0} .
\end{aligned}
$$

The constrained maximum likelihood estimates are solved as

$$
\begin{aligned}
\tilde{\mu} & =\psi_{0}-A \tilde{\sigma} \\
\tilde{\lambda} & =-l_{\mu}(\tilde{\theta}),
\end{aligned}
$$

where $\tilde{\sigma}$ satisfies

$$
-\frac{n}{\tilde{\sigma}}-\sum\left\{\frac{w_{i}-\psi_{0}}{\tilde{\sigma}^{2}}-\frac{w_{i}-\psi_{0}}{\tilde{\sigma}^{2}} \exp \left\{\frac{w_{i}-\psi_{0}}{\tilde{\sigma}}+A\right\}\right\}=0
$$


The tilted log-likelihood is

$$
\tilde{l}=l(\theta)+\tilde{\lambda}\left(\mu+\sigma A-\psi_{0}\right)
$$

with

$$
\begin{aligned}
\tilde{l}_{\mu}(\theta) & =l_{\mu}(\theta)+\tilde{\lambda} \\
\tilde{l}_{\sigma}(\theta) & =l_{\sigma}(\theta)+\tilde{\lambda} A \\
\tilde{l}_{\mu \mu}(\theta) & =l_{\mu \mu}(\theta) \\
\tilde{l}_{\mu \sigma}(\theta) & =l_{\mu \sigma}(\theta) \\
\tilde{l}_{\sigma \sigma}(\theta) & =l_{\sigma \sigma}(\theta) .
\end{aligned}
$$

The rest of the quantities are likewise the same as in Case 1 with $\psi_{\theta}(\theta)=\left[\begin{array}{ll}1 & A\end{array}\right]$.

As the maximum likelihood departure, $Q$, is explicitly provided in this paper, obtaining the thirdorder intervals becomes a programming matter. In the next section, results are provided for the $90 \%$, 95\%, and 99\% first- and third-order confidence intervals for Kennan's (1985) strike duration data. In addition, simulation results are recorded for the interest parameters discussed in the above three cases, specifically for the shape, scale, and median parameters. Results are provided for both the censored and complete data sets.

\section{Results}

For the empirical illustration, a sample of the strike duration data given in Kennan (1985) is used. The data used by Kennan is from the Bureau of Labor Statistics and reflects strike durations, in days, for the U.S. manufacturing industries for the 1968-1976 period. The complete and censored data sets considered are as in Kiefer (1988); the complete sample is restricted to strikes beginning in June of each year for a total number of 62 observations, the censored sample is formed by censoring durations greater than or equal to 80 days, resulting in 12 censored observations or approximately a $20 \%$ censored sample.

The first-order results were computed using Limdep. All third-order computations were done in Splus. In the tables below "lr" refers to the first-order likelihood ratio approach, "mle" refers to the first-order maximum likelihood approach, "LR" and "BN" refer to the third-order Lugannani and Rice and Barndorff-Nielsen approaches, respectively. 


\subsection{Censored Case}

\subsubsection{Confidence Intervals}

The resulting 90\%, 95\%, and 99\% confidence intervals are provided in Tables 1 to 3 below for the shape, scale, and median parameters. For the shape parameter, the confidence intervals resulting from using either the likelihood ratio or the mle are wider than the confidence intervals obtained from using the third-order methods. For the scale parameter, the first-order confidence intervals differ from the third-order ones predominantly on the upper tail, with the mle-based intervals noticeably discordant. Examination of the confidence intervals for the median reveals that the intervals based on the likelihood ratio are close to those based on the third-order methods, while the mle-based intervals are consistently very different.

Table 1: Confidence Intervals for Shape

\begin{tabular}{lcccccc}
\hline \multirow{2}{*}{ Method } & \multicolumn{2}{c}{$90 \%$ CI } & \multicolumn{2}{c}{$95 \%$ CI } & \multicolumn{2}{c}{$99 \%$ CI } \\
\cline { 2 - 7 } & lower & upper & lower & upper & lower & upper \\
\hline $\mathrm{lr}$ & 0.7254 & 1.0762 & 0.6959 & 1.1141 & 0.6403 & 1.1902 \\
mle & 0.6874 & 1.0943 & 0.6484 & 1.1333 & 0.5722 & 1.2095 \\
LR & 0.7085 & 1.0567 & 0.6793 & 1.0943 & 0.6243 & 1.1700 \\
BN & 0.7086 & 1.0568 & 0.6794 & 1.0944 & 0.6244 & 1.1701 \\
\hline
\end{tabular}

Table 2: Confidence Intervals for Scale

\begin{tabular}{lcccccc}
\hline \multirow{2}{*}{ Method } & \multicolumn{2}{c}{$90 \%$ CI } & \multicolumn{2}{c}{$95 \%$ CI } & \multicolumn{2}{c}{$99 \%$ CI } \\
\cline { 2 - 7 } & lower & upper & lower & upper & lower & upper \\
\hline lr & 32.2662 & 54.0480 & 30.6670 & 58.2784 & 27.7084 & 65.5739 \\
mle & 32.4231 & 54.0187 & 30.8765 & 56.7244 & 28.0621 & 62.4134 \\
LR & 32.4226 & 55.9020 & 30.7924 & 59.2698 & 27.7728 & 66.9105 \\
BN & 32.4226 & 55.9010 & 30.7924 & 59.2684 & 27.7728 & 66.9083 \\
\hline
\end{tabular}

Table 3: Confidence Intervals for the Median

\begin{tabular}{lcccccc}
\hline \multirow{2}{*}{ Method } & \multicolumn{2}{c}{$90 \%$ CI } & \multicolumn{2}{c}{$95 \%$ CI } & \multicolumn{2}{c}{$99 \%$ CI } \\
\cline { 2 - 7 } & lower & upper & lower & upper & lower & upper \\
\hline lr & 20.7006 & 36.5278 & 19.4879 & 38.5315 & 17.2222 & 42.8831 \\
mle & 20.6559 & 34.8133 & 19.3004 & 36.1688 & 16.6497 & 38.8195 \\
LR & 20.6409 & 36.8054 & 19.4097 & 38.8670 & 17.1119 & 43.3584 \\
BN & 20.6409 & 36.8053 & 19.4097 & 38.8668 & 17.1119 & 43.3581 \\
\hline
\end{tabular}

\subsubsection{Simulation}

To examine the accuracy of the censored case, a simulation study was conducted with 10,000 simulated samples. The shape and scale parameters were set equal to 1 . Two sample sizes $(n)$ with various 
censoring percentages were considered. Results are reported for the following cases:

\begin{tabular}{|c|c|c|}
\hline Case & $\mathrm{n}$ & Censored \% \\
\hline 1 & 10 & 10 \\
\hline 2 & 10 & 20 \\
\hline 3 & 10 & 30 \\
\hline 4 & 20 & 10 \\
\hline 5 & 20 & 20 \\
\hline 6 & 20 & 30 \\
\hline
\end{tabular}

For each simulated sample, only the $95 \%$ confidence intervals are reported. Tables 4 to 6 record the central coverage, coverage error, upper error, lower error, and average bias. The theoretical values for these quantities are $0.95,0.05,0.025,0.025$ and 0 , respectively. Central coverage is the proportion of intervals for which the true parameter value is within $95 \%$ confidence. Coverage error is defined as the proportion of intervals for which the true parameter value is outside $95 \%$ confidence. Upper and lower errors correspond to the proportion of intervals that fall outside the upper and lower confidence limits. Average bias is defined as (|Upper Error $-\alpha / 2|+|$ Lower Error $-\alpha / 2 \mid) / 2$, where $1-\alpha$ is the nominal coverage level.

From these tables it is evident that the confidence intervals based on third-order likelihood theory have excellent coverage properties. The conventional intervals based on the maximum likelihood estimate perform very poorly with highly asymmetric errors. For the shape parameter, holding the sample size constant, the average bias of the mle-based intervals increases as the censoring percentage increases. The likelihood ratio-based intervals improve on the mle-based ones in terms of central coverage, however, they nonetheless yield particularly asymmetric errors for the scale and median parameters. The third-order intervals possess the smallest average bias for all cases and parameters considered.

An additional simulation that matches the parameters of the strike duration data example more closely was considered. The simulation was based on 10,000 samples of 60 observations with a shape parameter set equal to 0.9 and a scale parameter set equal to 43 . The censoring percentage was set equal to 20. The results are recorded in Table 7. As can be seen from the table, for a sample size of 60 , the third-order method outperforms the other methods, especially for the shape parameter, in terms of average bias. 
Table 4: Simulation for Shape - Nominal: 95\%

\begin{tabular}{|c|c|c|c|c|c|c|}
\hline Case & Method & Coverage & Coverage Error & Upper Error & Lower Error & Average Bias \\
\hline & $\operatorname{lr}$ & 0.9502 & 0.0498 & 0.0264 & 0.0234 & 0.0015 \\
\hline \multirow[t]{3}{*}{1} & mle & 0.9318 & 0.0682 & 0.0004 & 0.0678 & 0.0337 \\
\hline & LR & 0.9511 & 0.0489 & 0.0253 & 0.0236 & 0.0009 \\
\hline & $\mathrm{BN}$ & 0.9504 & 0.0496 & 0.0244 & 0.0252 & 0.0004 \\
\hline & lr & 0.9510 & 0.0490 & 0.0242 & 0.0248 & 0.0005 \\
\hline \multirow[t]{3}{*}{2} & mle & 0.9195 & 0.0805 & 0.0001 & 0.0804 & 0.0402 \\
\hline & LR & 0.9498 & 0.0502 & 0.0256 & 0.0246 & 0.0005 \\
\hline & BN & 0.9499 & 0.0501 & 0.0248 & 0.0253 & 0.0003 \\
\hline \multirow{4}{*}{3} & $\operatorname{lr}$ & 0.9483 & 0.0517 & 0.0261 & 0.0256 & 0.0009 \\
\hline & mle & 0.9095 & 0.0905 & 0.0000 & 0.0905 & 0.0453 \\
\hline & LR & 0.9507 & 0.0493 & 0.0241 & 0.0252 & 0.0006 \\
\hline & $\mathrm{BN}$ & 0.9504 & 0.0496 & 0.0245 & 0.0251 & 0.0003 \\
\hline \multirow{4}{*}{4} & lr & 0.9515 & 0.0485 & 0.0268 & 0.0217 & 0.0026 \\
\hline & mle & 0.9398 & 0.0602 & 0.0036 & 0.0566 & 0.0265 \\
\hline & LR & 0.9498 & 0.0502 & 0.0258 & 0.0244 & 0.0007 \\
\hline & $\mathrm{BN}$ & 0.9506 & 0.0494 & 0.0248 & 0.0246 & 0.0003 \\
\hline \multirow{4}{*}{5} & lr & 0.9507 & 0.0493 & 0.0257 & 0.0236 & 0.0011 \\
\hline & mle & 0.9322 & 0.0678 & 0.0035 & 0.0643 & 0.0304 \\
\hline & LR & 0.9501 & 0.0499 & 0.0244 & 0.0255 & 0.0005 \\
\hline & BN & 0.9499 & 0.0501 & 0.0242 & 0.0259 & 0.0009 \\
\hline \multirow{4}{*}{6} & $\operatorname{lr}$ & 0.9479 & 0.0521 & 0.0254 & 0.0267 & 0.0011 \\
\hline & mle & 0.9298 & 0.0702 & 0.0019 & 0.0683 & 0.0332 \\
\hline & LR & 0.9492 & 0.0508 & 0.0247 & 0.0261 & 0.0007 \\
\hline & BN & 0.9498 & 0.0502 & 0.0249 & 0.0253 & 0.0002 \\
\hline
\end{tabular}


Table 5: Simulation for Scale - Nominal: 95\%

\begin{tabular}{|c|c|c|c|c|c|c|}
\hline Case & Method & Coverage & Coverage Error & Upper Error & Lower Error & Average Bias \\
\hline & lr & 0.9522 & 0.0478 & 0.0311 & 0.0167 & 0.0072 \\
\hline \multirow[t]{4}{*}{1} & mle & 0.9291 & 0.0709 & 0.0455 & 0.0254 & 0.0105 \\
\hline & $\mathrm{LR}$ & 0.9485 & 0.0515 & 0.0279 & 0.0236 & 0.0022 \\
\hline & $\mathrm{BN}$ & 0.9497 & 0.0503 & 0.0263 & 0.0240 & 0.0012 \\
\hline & $\operatorname{lr}$ & 0.9522 & 0.0478 & 0.0323 & 0.0155 & 0.0084 \\
\hline \multirow[t]{4}{*}{2} & mle & 0.9250 & 0.0750 & 0.0435 & 0.0315 & 0.0125 \\
\hline & $\mathrm{LR}$ & 0.9494 & 0.0506 & 0.0263 & 0.0243 & 0.0010 \\
\hline & $\mathrm{BN}$ & 0.9491 & 0.0509 & 0.0258 & 0.0251 & 0.0004 \\
\hline & $\operatorname{lr}$ & 0.9487 & 0.0513 & 0.0352 & 0.0161 & 0.0096 \\
\hline \multirow[t]{4}{*}{3} & mle & 0.9227 & 0.0773 & 0.0368 & 0.0405 & 0.0137 \\
\hline & LR & 0.9500 & 0.0500 & 0.0259 & 0.0241 & 0.0009 \\
\hline & $\mathrm{BN}$ & 0.9492 & 0.0508 & 0.0256 & 0.0252 & 0.0004 \\
\hline & $\operatorname{lr}$ & 0.9534 & 0.0466 & 0.0290 & 0.0176 & 0.0057 \\
\hline \multirow[t]{4}{*}{4} & mle & 0.9388 & 0.0612 & 0.0369 & 0.0243 & 0.0063 \\
\hline & $\mathrm{LR}$ & 0.9497 & 0.0503 & 0.0256 & 0.0247 & 0.0005 \\
\hline & $\mathrm{BN}$ & 0.9498 & 0.0502 & 0.0253 & 0.0249 & 0.0002 \\
\hline & $\operatorname{lr}$ & 0.9528 & 0.0472 & 0.0313 & 0.0159 & 0.0077 \\
\hline \multirow[t]{4}{*}{5} & mle & 0.9379 & 0.0621 & 0.0344 & 0.0277 & 0.0061 \\
\hline & $\mathrm{LR}$ & 0.9503 & 0.0497 & 0.0240 & 0.0257 & 0.0009 \\
\hline & $\mathrm{BN}$ & 0.9505 & 0.0495 & 0.0244 & 0.0251 & 0.0004 \\
\hline & lr & 0.9503 & 0.0497 & 0.0330 & 0.0167 & 0.0082 \\
\hline \multirow[t]{3}{*}{6} & mle & 0.9348 & 0.0652 & 0.0294 & 0.0358 & 0.0076 \\
\hline & LR & 0.9488 & 0.0512 & 0.0251 & 0.0261 & 0.0006 \\
\hline & $\mathrm{BN}$ & 0.9509 & 0.0491 & 0.0236 & 0.0255 & 0.0009 \\
\hline
\end{tabular}


Table 6: Simulation for Median - Nominal: 95\%

\begin{tabular}{|c|c|c|c|c|c|c|}
\hline Case & Method & Coverage & Coverage Error & Upper Error & Lower Error & Average Bias \\
\hline & lr & 0.9473 & 0.0527 & 0.0264 & 0.0234 & 0.0015 \\
\hline \multirow[t]{4}{*}{1} & mle & 0.9278 & 0.0722 & 0.0556 & 0.0166 & 0.0195 \\
\hline & LR & 0.9502 & 0.0498 & 0.0259 & 0.0239 & 0.0010 \\
\hline & $\mathrm{BN}$ & 0.9515 & 0.0485 & 0.0241 & 0.0244 & 0.0008 \\
\hline & lr & 0.9565 & 0.0435 & 0.0297 & 0.0138 & 0.0080 \\
\hline \multirow[t]{4}{*}{2} & mle & 0.9215 & 0.0785 & 0.0585 & 0.0200 & 0.0193 \\
\hline & LR & 0.9502 & 0.0498 & 0.0257 & 0.0241 & 0.0008 \\
\hline & $\mathrm{BN}$ & 0.9502 & 0.0498 & 0.0251 & 0.0247 & 0.0002 \\
\hline & $\operatorname{lr}$ & 0.9549 & 0.0451 & 0.0314 & 0.0137 & 0.0089 \\
\hline \multirow[t]{4}{*}{3} & mle & 0.9170 & 0.0830 & 0.0576 & 0.0254 & 0.0165 \\
\hline & LR & 0.9521 & 0.0479 & 0.0252 & 0.0227 & 0.0013 \\
\hline & $\mathrm{BN}$ & 0.9528 & 0.0472 & 0.0231 & 0.0241 & 0.0014 \\
\hline & lr & 0.9542 & 0.0458 & 0.0278 & 0.0180 & 0.0049 \\
\hline \multirow[t]{4}{*}{4} & mle & 0.9398 & 0.0602 & 0.0446 & 0.0156 & 0.0145 \\
\hline & LR & 0.9499 & 0.0501 & 0.0262 & 0.0239 & 0.0012 \\
\hline & $\mathrm{BN}$ & 0.9510 & 0.0490 & 0.0244 & 0.0246 & 0.0005 \\
\hline & lr & 0.9546 & 0.0454 & 0.0290 & 0.0164 & 0.0063 \\
\hline \multirow[t]{4}{*}{5} & mle & 0.9356 & 0.0644 & 0.0470 & 0.0174 & 0.0148 \\
\hline & LR & 0.9502 & 0.0498 & 0.0257 & 0.0241 & 0.0008 \\
\hline & $\mathrm{BN}$ & 0.9496 & 0.0504 & 0.0262 & 0.0242 & 0.0010 \\
\hline & $\operatorname{lr}$ & 0.9536 & 0.0464 & 0.0306 & 0.0158 & 0.0074 \\
\hline \multirow[t]{3}{*}{6} & mle & 0.9296 & 0.0704 & 0.0453 & 0.0251 & 0.0102 \\
\hline & $\mathrm{LR}$ & 0.9492 & 0.0508 & 0.0251 & 0.0257 & 0.0004 \\
\hline & $\mathrm{BN}$ & 0.9504 & 0.0496 & 0.0244 & 0.0252 & 0.0004 \\
\hline
\end{tabular}

Table 7: Simulation $(n=60$, shape $=0.9$, scale $=43$, censored $\%=20)$ - Nominal: $95 \%$

\begin{tabular}{ccccccc}
\hline Parameter & Method & Coverage & Coverage Error & Upper Error & Lower Error & Average Bias \\
\hline \multirow{5}{*}{ Shape } & lr & 0.9499 & 0.0501 & 0.0229 & 0.0272 & 0.0022 \\
& mle & 0.9652 & 0.0348 & 0.0197 & 0.0151 & 0.0076 \\
& LR & 0.9502 & 0.0498 & 0.0245 & 0.0253 & 0.0004 \\
& BN & 0.9501 & 0.0499 & 0.0245 & 0.0254 & 0.0004 \\
\hline \multirow{3}{*}{ Scale } & lr & 0.9505 & 0.0495 & 0.0260 & 0.0235 & 0.0013 \\
& mle & 0.9438 & 0.0562 & 0.0299 & 0.0263 & 0.0031 \\
& LR & 0.9501 & 0.0499 & 0.0258 & 0.0241 & 0.0009 \\
& BN & 0.9501 & 0.0499 & 0.0258 & 0.0241 & 0.0009 \\
\hline \multirow{3}{*}{ Median } & lr & 0.9494 & 0.0506 & 0.0259 & 0.0247 & 0.0006 \\
& mle & 0.9444 & 0.0556 & 0.0302 & 0.0254 & 0.0028 \\
& LR & 0.9494 & 0.0506 & 0.0255 & 0.0251 & 0.0003 \\
& BN & 0.9495 & 0.0505 & 0.0255 & 0.0250 & 0.0002 \\
\hline
\end{tabular}




\subsection{Complete Case}

\subsubsection{Confidence Intervals}

The resulting 90\%, 95\%, and 99\% confidence intervals for the complete (uncensored) strike duration data are provided in Tables 8 to 10 below for the shape, scale, and median parameters. For the shape parameter, the confidence intervals resulting from using either the likelihood ratio or the mle are again wider than the confidence intervals obtained from using the third-order methods. For the scale and median parameters, the likelihood ratio-based confidence intervals are close to the third-order ones, the mle-based intervals are, however, very discordant especially for the median.

Table 8: Confidence Intervals for Shape

\begin{tabular}{lcccccc}
\hline \multirow{2}{*}{ Method } & \multicolumn{2}{c}{$90 \%$ CI } & \multicolumn{2}{c}{$95 \%$ CI } & \multicolumn{2}{c}{$99 \%$ CI } \\
\cline { 2 - 7 } & lower & upper & lower & upper & lower & upper \\
\hline lr & 0.7804 & 1.0816 & 0.7542 & 1.1131 & 0.7043 & 1.1760 \\
mle & 0.7407 & 1.1087 & 0.7055 & 1.1439 & 0.6366 & 1.2128 \\
LR & 0.7675 & 1.0675 & 0.7414 & 1.0989 & 0.6918 & 1.1615 \\
BN & 0.7675 & 1.0675 & 0.7415 & 1.0989 & 0.6918 & 1.1616 \\
\hline
\end{tabular}

Table 9: Confidence Intervals for Scale

\begin{tabular}{lcccccc}
\hline \multirow{2}{*}{ Method } & \multicolumn{2}{c}{$90 \%$ CI } & \multicolumn{2}{c}{$95 \%$ CI } & \multicolumn{2}{c}{$99 \%$ CI } \\
\cline { 2 - 7 } & lower & upper & lower & upper & lower & upper \\
\hline lr & 32.1673 & 52.1166 & 30.6225 & 54.5717 & 27.7333 & 59.8118 \\
mle & 32.4088 & 52.1420 & 30.9663 & 54.5709 & 28.3283 & 59.6526 \\
LR & 32.1757 & 52.4719 & 30.6111 & 54.9834 & 27.6871 & 60.3551 \\
BN & 32.1757 & 52.4721 & 30.6111 & 54.9833 & 27.6871 & 60.3548 \\
\hline
\end{tabular}

Table 10: Confidence Intervals for the Median

\begin{tabular}{lcccccc}
\hline \multirow{2}{*}{ Method } & \multicolumn{2}{c}{$90 \%$ CI } & \multicolumn{2}{c}{$95 \%$ CI } & \multicolumn{2}{c}{$99 \%$ CI } \\
\cline { 2 - 7 } & lower & upper & lower & upper & lower & upper \\
\hline lr & 20.8600 & 35.7189 & 19.6778 & 37.4504 & 17.4700 & 41.0558 \\
mle & 21.0801 & 34.2316 & 19.8209 & 35.4908 & 17.3585 & 37.9532 \\
LR & 20.7370 & 35.7720 & 19.5452 & 37.5311 & 17.3212 & 41.1991 \\
BN & 20.7370 & 35.7720 & 19.5452 & 37.5311 & 17.3213 & 41.1991 \\
\hline
\end{tabular}

\subsubsection{Simulation}

In this section simulation results for the complete data case for 10,000 simulated samples are reported. The shape parameter took on the values 1, 0.5 and 5 , and the scale parameter took on the values 1 and 10. Sample sizes $(n)$ of size 5 and 10 were considered. Results are reported for the following 12 cases: 
Simulation Settings

\begin{tabular}{cccc}
\hline Case & $\mathrm{n}$ & shape & scale \\
\hline 1 & 5 & 1 & 1 \\
2 & 10 & 1 & 1 \\
3 & 5 & 1 & 10 \\
4 & 10 & 1 & 10 \\
5 & 5 & 0.5 & 1 \\
6 & 10 & 0.5 & 1 \\
7 & 5 & 0.5 & 10 \\
8 & 10 & 0.5 & 10 \\
9 & 5 & 5 & 1 \\
10 & 10 & 5 & 1 \\
11 & 5 & 5 & 10 \\
12 & 10 & 5 & 10 \\
\hline
\end{tabular}

For each simulated sample, only the $95 \%$ confidence intervals are reported. Tables 11 to 13 record the central coverage, coverage error, upper error, lower error, and average bias. The simulation results for the complete case illustrate the dominance of the third-order approximations. For every case considered, the average bias is again smallest for these third-order intervals compared to the first-order intervals. Further, this bias decreases for any given shape and scale combination as the sample size increases from 5 to 10. The intervals based on the maximum likelihood estimates perform very poorly and produce highly asymmetric errors. The likelihood ratio-based intervals produce highly asymmetric error rates as well, although in terms of central coverage they do outperform the mle-based intervals.

An additional simulation similar to that produced in Table 7 was considered. The simulation was based on 10,000 samples of 60 observations with a shape parameter set equal to 0.9 and a scale parameter set equal to 43. The results are recorded in Table 14. The third-order method generally outperforms the other methods in terms of central coverage and average bias even for this sample size of 60. Again, the largest gain, in terms of bias, from using the third-order method arises for the shape parameter. 
Table 11: Simulation for Shape - Nominal: 95\%

\begin{tabular}{|c|c|c|c|c|c|c|}
\hline Case & Method & Coverage & Coverage Error & Upper Error & Lower Error & Average Bias \\
\hline & lr & 0.9465 & 0.0535 & 0.0323 & 0.0212 & 0.0056 \\
\hline \multirow[t]{3}{*}{1} & mle & 0.9582 & 0.0418 & 0.0094 & 0.0324 & 0.0115 \\
\hline & LR & 0.9490 & 0.0510 & 0.0275 & 0.0235 & 0.0020 \\
\hline & $\mathrm{BN}$ & 0.9492 & 0.0508 & 0.0289 & 0.0219 & 0.0035 \\
\hline \multirow{4}{*}{2} & $\operatorname{lr}$ & 0.9423 & 0.0577 & 0.0309 & 0.0268 & 0.0039 \\
\hline & mle & 0.9369 & 0.0631 & 0.0112 & 0.0519 & 0.0204 \\
\hline & LR & 0.9500 & 0.0500 & 0.0260 & 0.0240 & 0.0010 \\
\hline & $\mathrm{BN}$ & 0.9499 & 0.0501 & 0.0263 & 0.0238 & 0.0013 \\
\hline \multirow{4}{*}{3} & lr & 0.9440 & 0.0560 & 0.0328 & 0.0232 & 0.0048 \\
\hline & mle & 0.9386 & 0.0614 & 0.0169 & 0.0445 & 0.0138 \\
\hline & LR & 0.9490 & 0.0510 & 0.0275 & 0.0235 & 0.0020 \\
\hline & $\mathrm{BN}$ & 0.9492 & 0.0508 & 0.0289 & 0.0219 & 0.0035 \\
\hline \multirow{4}{*}{4} & lr & 0.9471 & 0.0529 & 0.0288 & 0.0241 & 0.0024 \\
\hline & mle & 0.9474 & 0.0526 & 0.0227 & 0.0299 & 0.0036 \\
\hline & LR & 0.9500 & 0.0500 & 0.0260 & 0.0240 & 0.0010 \\
\hline & $\mathrm{BN}$ & 0.9499 & 0.0501 & 0.0263 & 0.0238 & 0.0013 \\
\hline \multirow{4}{*}{5} & lr & 0.9504 & 0.0496 & 0.0298 & 0.0198 & 0.0050 \\
\hline & mle & 0.9471 & 0.0529 & 0.0192 & 0.0337 & 0.0073 \\
\hline & LR & 0.9488 & 0.0512 & 0.0277 & 0.0235 & 0.0021 \\
\hline & BN & 0.9491 & 0.0509 & 0.0290 & 0.0219 & 0.0036 \\
\hline \multirow{4}{*}{6} & lr & 0.9551 & 0.0449 & 0.0186 & 0.0263 & 0.0039 \\
\hline & mle & 0.9533 & 0.0467 & 0.0295 & 0.0172 & 0.0062 \\
\hline & LR & 0.9502 & 0.0498 & 0.0260 & 0.0238 & 0.0011 \\
\hline & $\mathrm{BN}$ & 0.9501 & 0.0499 & 0.0263 & 0.0236 & 0.0014 \\
\hline \multirow{4}{*}{7} & $\operatorname{lr}$ & 0.9406 & 0.0594 & $\begin{array}{l}0.0324 \\
\end{array}$ & 0.0270 & 0.0047 \\
\hline & mle & 0.9607 & 0.0393 & 0.0169 & 0.0224 & 0.0054 \\
\hline & LR & 0.9488 & 0.0512 & 0.0277 & 0.0235 & 0.0021 \\
\hline & $\mathrm{BN}$ & 0.9491 & 0.0509 & 0.0290 & 0.0219 & 0.0036 \\
\hline \multirow{4}{*}{8} & lr & 0.9447 & 0.0553 & 0.0244 & 0.0309 & 0.0033 \\
\hline & mle & 0.9394 & 0.0606 & 0.0205 & 0.0401 & 0.0098 \\
\hline & LR & 0.9502 & 0.0498 & 0.0260 & 0.0238 & 0.0011 \\
\hline & $\mathrm{BN}$ & 0.9501 & 0.0499 & 0.0263 & 0.0236 & 0.0014 \\
\hline \multirow{4}{*}{9} & lr & 0.9435 & 0.0565 & 0.0362 & 0.0203 & 0.0080 \\
\hline & mle & 0.9399 & 0.0601 & 0.0099 & 0.0502 & 0.0202 \\
\hline & LR & 0.9490 & 0.0510 & 0.0275 & 0.0235 & 0.0020 \\
\hline & $\mathrm{BN}$ & 0.9492 & 0.0508 & 0.0289 & 0.0219 & 0.0035 \\
\hline \multirow{4}{*}{10} & $\operatorname{lr}$ & 0.9502 & 0.0498 & 0.0326 & 0.0172 & 0.0077 \\
\hline & mle & 0.9318 & 0.0682 & 0.0141 & 0.0541 & 0.0200 \\
\hline & LR & 0.9499 & 0.0501 & 0.0261 & 0.0240 & 0.0011 \\
\hline & $\mathrm{BN}$ & 0.9500 & 0.0500 & 0.0264 & 0.0236 & 0.0014 \\
\hline \multirow{4}{*}{11} & lr & 0.9494 & 0.0506 & 0.0315 & 0.0191 & 0.0062 \\
\hline & mle & 0.9406 & 0.0594 & 0.0057 & 0.0537 & 0.0240 \\
\hline & LR & 0.9490 & 0.0510 & 0.0275 & 0.0235 & 0.0020 \\
\hline & $\mathrm{BN}$ & 0.9492 & 0.0508 & 0.0289 & 0.0219 & 0.0035 \\
\hline \multirow{4}{*}{12} & lr & 0.9452 & 0.0548 & 0.0237 & 0.0311 & 0.0037 \\
\hline & mle & 0.9346 & 0.0654 & 0.0192 & 0.0462 & 0.0135 \\
\hline & LR & 0.9499 & 0.0501 & 0.0261 & 0.0240 & 0.0011 \\
\hline & $\mathrm{BN}$ & 0.9500 & 0.0500 & 0.0264 & 0.0236 & 0.0014 \\
\hline
\end{tabular}


Table 12: Simulation for Scale - Nominal: 95\%

\begin{tabular}{|c|c|c|c|c|c|c|}
\hline Case & Method & Coverage & Coverage Error & Upper Error & Lower Error & Average Bias \\
\hline & $\operatorname{lr}$ & 0.9443 & 0.0557 & 0.0317 & 0.0240 & 0.0039 \\
\hline \multirow[t]{3}{*}{1} & mle & 0.9171 & 0.0829 & 0.0530 & 0.0299 & 0.0165 \\
\hline & LR & 0.9480 & 0.0520 & 0.0261 & 0.0259 & 0.0010 \\
\hline & $\mathrm{BN}$ & 0.9468 & 0.0532 & 0.0272 & 0.0260 & 0.0016 \\
\hline \multirow{4}{*}{2} & $\operatorname{lr}$ & 0.9500 & 0.0500 & 0.0264 & 0.0236 & 0.0014 \\
\hline & mle & 0.9387 & 0.0613 & 0.0330 & 0.0283 & 0.0057 \\
\hline & LR & 0.9497 & 0.0503 & 0.0258 & 0.0245 & 0.0007 \\
\hline & $\mathrm{BN}$ & 0.9495 & 0.0505 & 0.0261 & 0.0244 & 0.0009 \\
\hline \multirow{4}{*}{3} & $\operatorname{lr}$ & 0.9439 & 0.0561 & 0.0326 & 0.0235 & 0.0046 \\
\hline & mle & 0.9169 & 0.0831 & 0.0472 & 0.0359 & 0.0166 \\
\hline & LR & 0.9480 & 0.0520 & 0.0261 & 0.0259 & 0.0010 \\
\hline & BN & 0.9468 & 0.0532 & 0.0272 & 0.0260 & 0.0016 \\
\hline \multirow{4}{*}{4} & $\operatorname{lr}$ & 0.9491 & 0.0509 & 0.0311 & 0.0198 & 0.0057 \\
\hline & mle & 0.9259 & 0.0741 & 0.0413 & 0.0328 & 0.0121 \\
\hline & LR & 0.9497 & 0.0503 & 0.0258 & 0.0245 & 0.0007 \\
\hline & $\mathrm{BN}$ & 0.9495 & 0.0505 & 0.0261 & 0.0244 & 0.0009 \\
\hline \multirow{4}{*}{5} & $\operatorname{lr}$ & 0.9440 & 0.0560 & 0.0359 & 0.0201 & 0.0079 \\
\hline & mle & 0.9179 & 0.0821 & 0.0507 & 0.0314 & 0.0161 \\
\hline & LR & 0.9477 & 0.0523 & 0.0264 & 0.0259 & 0.0012 \\
\hline & $\mathrm{BN}$ & 0.9466 & 0.0534 & 0.0274 & 0.0260 & 0.0017 \\
\hline \multirow{4}{*}{6} & lr & 0.9439 & 0.0561 & 0.0323 & 0.0238 & 0.0043 \\
\hline & mle & 0.9365 & 0.0635 & 0.0362 & 0.0273 & 0.0068 \\
\hline & LR & 0.9496 & 0.0504 & 0.0259 & 0.0245 & 0.0007 \\
\hline & BN & 0.9494 & 0.0506 & 0.0262 & 0.0244 & 0.0009 \\
\hline \multirow{4}{*}{7} & $\operatorname{lr}$ & 0.9470 & 0.0530 & 0.0299 & 0.0231 & 0.0034 \\
\hline & mle & 0.9409 & 0.0591 & 0.0343 & 0.0248 & 0.0048 \\
\hline & LR & 0.9477 & 0.0523 & 0.0264 & 0.0259 & 0.0012 \\
\hline & $\mathrm{BN}$ & 0.9466 & 0.0534 & 0.0274 & 0.0260 & 0.0017 \\
\hline \multirow{4}{*}{8} & lr & 0.9502 & 0.0498 & 0.0305 & 0.0193 & 0.0056 \\
\hline & mle & 0.9435 & 0.0565 & 0.0370 & 0.0195 & 0.0088 \\
\hline & LR & 0.9496 & 0.0504 & 0.0259 & 0.0245 & 0.0007 \\
\hline & $\mathrm{BN}$ & 0.9494 & 0.0506 & 0.0262 & 0.0244 & 0.0009 \\
\hline \multirow{4}{*}{9} & $\operatorname{lr}$ & 0.9514 & 0.0486 & 0.0277 & 0.0209 & 0.0034 \\
\hline & mle & 0.9605 & 0.0395 & 0.0297 & 0.0098 & 0.0100 \\
\hline & LR & 0.9478 & 0.0522 & 0.0262 & 0.0260 & 0.0011 \\
\hline & $\mathrm{BN}$ & 0.9467 & 0.0533 & 0.0273 & 0.0260 & 0.0017 \\
\hline \multirow{4}{*}{10} & lr & 0.9495 & 0.0505 & 0.0303 & 0.0202 & 0.0051 \\
\hline & mle & 0.9487 & 0.0513 & 0.0314 & 0.0199 & 0.0058 \\
\hline & LR & 0.9496 & 0.0504 & 0.0258 & 0.0246 & 0.0006 \\
\hline & $\mathrm{BN}$ & 0.9494 & 0.0506 & 0.0261 & 0.0245 & 0.0008 \\
\hline \multirow{4}{*}{11} & lr & 0.9396 & 0.0604 & 0.0341 & 0.0263 & 0.0052 \\
\hline & mle & 0.9281 & 0.0719 & 0.0401 & 0.0318 & 0.0110 \\
\hline & LR & 0.9478 & 0.0522 & 0.0262 & 0.0260 & 0.0011 \\
\hline & BN & 0.9467 & 0.0533 & 0.0273 & 0.0260 & 0.0017 \\
\hline \multirow{4}{*}{12} & $\mathrm{lr}$ & 0.9479 & 0.0521 & 0.0337 & 0.0184 & 0.0077 \\
\hline & mle & 0.9266 & 0.0734 & 0.0428 & 0.0306 & 0.0117 \\
\hline & LR & 0.9496 & 0.0504 & 0.0258 & 0.0246 & 0.0006 \\
\hline & BN & 0.9494 & 0.0506 & 0.0261 & 0.0245 & 0.0008 \\
\hline
\end{tabular}


Table 13: Simulation for Median - Nominal: 95\%

\begin{tabular}{|c|c|c|c|c|c|c|}
\hline Case & Method & Coverage & Coverage Error & Upper Error & Lower Error & Average Bias \\
\hline & $\operatorname{lr}$ & 0.9562 & 0.0438 & 0.0267 & 0.0171 & 0.0048 \\
\hline \multirow[t]{3}{*}{1} & mle & 0.9530 & 0.0470 & 0.0299 & 0.0171 & 0.0064 \\
\hline & LR & 0.9483 & 0.0517 & 0.0250 & 0.0267 & 0.0009 \\
\hline & $\mathrm{BN}$ & 0.9482 & 0.0518 & 0.0249 & 0.0269 & 0.0010 \\
\hline \multirow{4}{*}{2} & lr & 0.9534 & 0.0466 & 0.0265 & 0.0201 & 0.0032 \\
\hline & mle & 0.9542 & 0.0458 & 0.0277 & 0.0181 & 0.0048 \\
\hline & LR & 0.9485 & 0.0515 & 0.0261 & 0.0254 & 0.0007 \\
\hline & $\mathrm{BN}$ & 0.9485 & 0.0515 & 0.0261 & 0.0254 & 0.0007 \\
\hline \multirow{4}{*}{3} & $\operatorname{lr}$ & 0.9500 & 0.0500 & 0.0304 & 0.0196 & 0.0054 \\
\hline & mle & 0.9498 & 0.0502 & 0.0313 & 0.0189 & 0.0062 \\
\hline & LR & 0.9483 & 0.0517 & 0.0250 & 0.0267 & 0.0009 \\
\hline & BN & 0.9482 & 0.0518 & 0.0249 & 0.0269 & 0.0010 \\
\hline \multirow{4}{*}{4} & lr & 0.9535 & 0.0465 & 0.0266 & 0.0199 & 0.0034 \\
\hline & mle & 0.9529 & 0.0471 & 0.0279 & 0.0192 & 0.0044 \\
\hline & LR & 0.9485 & 0.0515 & 0.0261 & 0.0254 & 0.0007 \\
\hline & $\mathrm{BN}$ & 0.9485 & 0.0515 & 0.0261 & 0.0254 & 0.0007 \\
\hline \multirow{4}{*}{5} & lr & 0.9581 & 0.0419 & 0.0245 & 0.0174 & 0.0041 \\
\hline & mle & 0.9501 & 0.0499 & 0.0212 & 0.0287 & 0.0038 \\
\hline & LR & 0.9482 & 0.0518 & 0.0250 & 0.0268 & 0.0009 \\
\hline & $\mathrm{BN}$ & 0.9481 & 0.0519 & 0.0250 & 0.0269 & 0.0009 \\
\hline \multirow{4}{*}{6} & $\operatorname{lr}$ & 0.9521 & 0.0479 & 0.0269 & 0.0210 & 0.0030 \\
\hline & mle & 0.9500 & 0.0500 & 0.0302 & 0.0198 & 0.0052 \\
\hline & LR & 0.9486 & 0.0514 & 0.0261 & 0.0253 & 0.0007 \\
\hline & BN & 0.9485 & 0.0515 & 0.0262 & 0.0253 & 0.0007 \\
\hline \multirow{4}{*}{7} & $\mathrm{lr}$ & 0.9526 & 0.0474 & 0.0307 & 0.0167 & 0.0070 \\
\hline & mle & 0.9538 & 0.0462 & 0.0322 & 0.0140 & 0.0091 \\
\hline & LR & 0.9482 & 0.0518 & 0.0250 & 0.0268 & 0.0009 \\
\hline & $\mathrm{BN}$ & 0.9481 & 0.0519 & 0.0250 & 0.0269 & 0.0009 \\
\hline \multirow{4}{*}{8} & lr & 0.9489 & 0.0511 & 0.0280 & 0.0231 & 0.0025 \\
\hline & mle & 0.9492 & 0.0508 & 0.0287 & 0.0221 & 0.0033 \\
\hline & LR & 0.9486 & 0.0514 & 0.0261 & 0.0253 & 0.0007 \\
\hline & $\mathrm{BN}$ & 0.9485 & 0.0515 & 0.0262 & 0.0253 & 0.0007 \\
\hline \multirow{4}{*}{9} & $\operatorname{lr}$ & 0.9496 & 0.0504 & 0.0313 & 0.0191 & 0.0061 \\
\hline & mle & 0.9536 & 0.0464 & 0.0345 & 0.0119 & 0.0113 \\
\hline & LR & 0.9483 & 0.0517 & 0.0251 & 0.0266 & 0.0008 \\
\hline & $\mathrm{BN}$ & 0.9481 & 0.0519 & 0.0251 & 0.0268 & 0.0009 \\
\hline \multirow{4}{*}{10} & lr & 0.9509 & 0.0491 & 0.0299 & 0.0192 & 0.0054 \\
\hline & mle & 0.9534 & 0.0466 & 0.0313 & 0.0153 & 0.0080 \\
\hline & LR & 0.9486 & 0.0514 & 0.0261 & 0.0252 & 0.0007 \\
\hline & $\mathrm{BN}$ & 0.9485 & 0.0515 & 0.0262 & 0.0251 & 0.0007 \\
\hline \multirow{4}{*}{11} & lr & 0.9466 & 0.0534 & 0.0316 & 0.0218 & 0.0049 \\
\hline & mle & 0.9555 & 0.0445 & 0.0347 & 0.0098 & 0.0125 \\
\hline & LR & 0.9483 & 0.0517 & 0.0251 & 0.0266 & 0.0008 \\
\hline & BN & 0.9481 & 0.0519 & 0.0251 & 0.0268 & 0.0009 \\
\hline \multirow{4}{*}{12} & $\operatorname{lr}$ & 0.9489 & 0.0511 & 0.0285 & 0.0226 & 0.0030 \\
\hline & mle & 0.9504 & 0.0496 & 0.0294 & 0.0202 & 0.0046 \\
\hline & LR & 0.9486 & 0.0514 & 0.0261 & 0.0252 & 0.0007 \\
\hline & BN & 0.9485 & 0.0515 & 0.0262 & 0.0251 & 0.0007 \\
\hline
\end{tabular}


Table 14: Simulation $(n=60$, shape $=0.9$, scale $=43)$ - Nominal: $95 \%$

\begin{tabular}{ccccccc}
\hline Parameter & Method & Coverage & Coverage Error & Upper Error & Lower Error & Average Bias \\
\hline \multirow{5}{*}{ Shape } & lr & 0.9496 & 0.0504 & 0.0231 & 0.0273 & 0.0021 \\
& mle & 0.9631 & 0.0369 & 0.0202 & 0.0167 & 0.0066 \\
& LR & 0.9500 & 0.0500 & 0.0247 & 0.0253 & 0.0003 \\
& BN & 0.9501 & 0.0499 & 0.0247 & 0.0252 & 0.0003 \\
\hline \multirow{3}{*}{ Scale } & lr & 0.9498 & 0.0502 & 0.0264 & 0.0238 & 0.0013 \\
& mle & 0.9419 & 0.0581 & 0.0319 & 0.0262 & 0.0041 \\
& LR & 0.9507 & 0.0493 & 0.0252 & 0.0241 & 0.0006 \\
& BN & 0.9508 & 0.0492 & 0.0252 & 0.0240 & 0.0006 \\
\hline \multirow{3}{*}{ Median } & lr & 0.9480 & 0.0520 & 0.0268 & 0.0252 & 0.0010 \\
& mle & 0.9422 & 0.0578 & 0.0321 & 0.0257 & 0.0039 \\
& LR & 0.9506 & 0.0494 & 0.0253 & 0.0241 & 0.0006 \\
& BN & 0.9508 & 0.0492 & 0.0252 & 0.0240 & 0.0006 \\
\hline
\end{tabular}

\section{Conclusion}

Recent third-order likelihood theory was applied to obtain highly accurate confidence intervals for the shape, scale, and median parameters of the Weibull distribution. Both the censored and complete cases were examined and applied to Kennan's (1985) strike duration data. The intervals obtained using the first-order quantities, namely, the likelihood ratio and the maximum likelihood estimate were considerably different from those intervals obtained using the third-order methods. The accuracy of the third-order intervals was assessed through simulations. For both the complete and censored cases, the simulations revealed that a significant improvement could be attained using the third-order-based confidence intervals. As stated in Section 3, the extra computation required for the third-order intervals is due to the calculation of the maximum departure measure, $Q$. However, the explicit equations for this quantity are provided in the paper. It is further noted that the calculation of $Q$ does not depend on the sample size but on the dimension of the parameter, and in this case the dimension is two, which makes the extra calculations minimal. On the other hand, the calculation of the maximum likelihood estimate and constrained maximum likelihood estimate depends on the sample size. Hence, for simulations, when the sample size is large, even the first-order methods will require considerable computational costs. In terms of the first-order methods, the likelihood ratio intervals performed relatively well and are the preferred method to the mle-based intervals. With respect to future research, it will be interesting to pursue these methods to Weibull problems that include regressors and hence more parameters.. 


\section{Acknowledgements}

The authors would like to thank D.A.S Fraser, an associate editor, and a referee for helpful comments.

Wong gratefully acknowledges the support of the Natural Sciences and Engineering Research Council of Canada. 


\section{References}

[1] Barndorff-Nielsen, O.E., 1991. Modified Signed Log-Likelihood Ratio. Biometrika 78, 557-563.

[2] Fraser, D.A.S., Reid, N., 1995. Ancillaries and Third-Order Significance. Utilitas Mathematica $7,33-55$.

[3] Fraser, D.A.S., Reid, N. Wu, J., 1999. A Simple General Formula for Tail Probabilities for Frequentist and Bayesian Inference. Biometrika 86, 249-264.

[4] Kennan, J., 1985. The Duration of Contract Strikes in U.S. Manufacturing. Journal of Econometrics $28,5-28$.

[5] Kiefer, N., 1988. Economic Duration Data and Hazard Funtions. Journal of Economic Literature $26,646-679$.

[6] Lawless, J.F., 1982. Statistical Models and Methods for Lifetime Data, John Wiley and Sons, New York.

[7] Lugannani, R., Rice, S.O., 1980. Saddlepoint Approximation for the Distribution of the Sums of Independent Random Variables. Advances in Applied Probability 12, 475-490. 